\title{
PET/MRI and the revolution of the third eye
}

\author{
Luigi Mansi • Andrea Ciarmiello • Vincenzo Cuccurullo
}

Published online: 17 July 2012

(C) Springer-Verlag 2012

\section{Introduction}

The 20th century diagnostic imaging scenario almost for the whole of its existence was divided into two well-separated universes: (1) the morphostructural, in which information on anatomy and structures are acquired, with pathology as the gold standard, and (2) the functional, in which normal and altered functions are analysed, with pathophysiology as the reference.

Morphostructural techniques show differences in density, as observed in a picture taken in a steady state as a freezeframe effectively not allowing distinction between a living and a dead body. Functional techniques, which can only be applied in a living being, show dynamic changes as in a movie where "time" is a major element, taking information from parameters such as differences in concentration and movement. This category includes techniques such as urography and cardio-CT, in which increasing doses in dynamic scans and the number of acquisitions are important considerations [1]. To avoid a high radiation dose, the lowest number of frames are acquired, and this is a major disadvantage with respect to nuclear medicine and MRI, which are queens in "clinical" functional imaging because of their superiority in studying pathophysiology.

L. Mansi $\cdot$ V. Cuccurullo

Nuclear Medicine Division, Second University of Naples, Naples, Italy

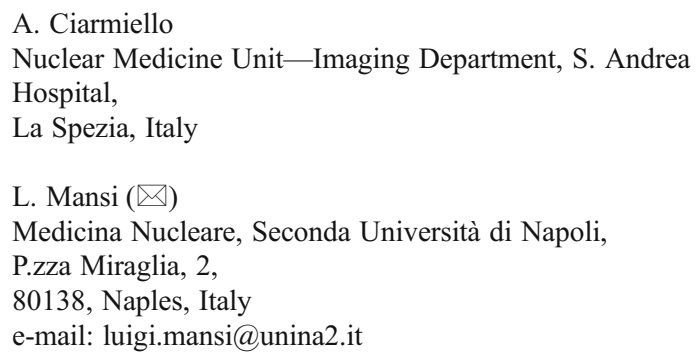

A. Ciarmiello

Nuclear Medicine Unit-Imaging Department, S. Andrea

Hospital,

La Spezia, Italy

L. Mansi $(\bowtie)$

Medicina Nucleare, Seconda Università di Napoli,

P.zza Miraglia, 2,

80138, Naples, Italy

e-mail: luigi.mansi@unina2.it

\section{Nuclear medicine as molecular imaging}

Nuclear medicine is different from other imaging techniques, being the only one possible exclusively as a functional method. Having as the goal the analysis of possible innovations allowed by PET/MRI, we centre our attention on the value of nuclear medicine in the context of molecular imaging.

Humans, as all living organisms, are made of biomolecules. Health is maintained through a dynamic equilibrium called homeostasis, which is controlled by regulation mechanisms. Many diseases result from disturbance of homeostasis, leading to imbalance in a molecular system. Starting from these premises, it is possible to diagnose an altered state by following biomolecular kinetics in normal (physiological) conditions and in illness (pathophysiological conditions).

To achieve this, for each system we need tracers that follow the kinetics of the native molecules. When labelled with a tag allowing detection by an outside scanner, these tracers become a diagnostic tool in molecular imaging. To image a normal or altered system, i.e. an environment where specific molecules are connected through a dynamic interaction, we should not influence the process. Therefore, reliable results may only be achieved when a picture can be produced with a very low number of tracer molecules with respect to the native ones involved in the system. Starting from this premise, today molecular imaging can be applied in most cases only in nuclear medicine and optical imaging, both of which have the ability to produce images with pico/nanomolar amounts of tracer. Conversely, MRI (and CT) may trace processes only using micro/millimolar amounts, i.e. too high to permit rigorous and harmless evaluation. Optical imaging, because it is based on light as the detectable signal, cannot be used to analyse deep structures. Thus, since the first radionuclide studies in the 1940s in thyroid diseases with ${ }^{131} \mathrm{I}$, tracing the presence of the iodine symporter gene in normal and well-differentiated 
neoplastic cells, clinical molecular imaging in humans has been almost a synonym for nuclear medicine.

Molecular imaging is dependent on the radiotracer more than on the scanner, so that planar scintigraphy, as in Meckel's diverticulum, is also cost-effective, although PET is preferable when suitable radiotracers are available. The value of PET lies in the fact that important biomolecules can be labelled with positron emitters. The most important radiotracers used in clinical practice today [2] include, together with ${ }^{18} \mathrm{~F}$ Fluorodeoxy-glucose (FDG), radiocholine used in restaging of prostate cancer, radioamino acids used to detect cerebral recurrences, radiopeptides used to evaluate neuroendocrine tumours, and ${ }^{18} \mathrm{~F}$ fluoride used for the early detection of bone metastases [3]. Newer applications are entering the clinical field, allowing the evaluation of growth rate using radiolabelled thymidine or the analysis of in vivo distribution of oestrogen receptors. The imaging of hypoxia and neoangiogenesis [4] and the study of brain diseases using radiotracers for amyloid, neurotransmitters and markers of neuroinflammation [5] are already feasible in humans, and a large number of research studies based on tracers of the most intriguing molecular mechanisms defining diseases at the level of genomics and proteomics are underway.

Given the establishment of the value of functional data as well as morphostructural data in hybrid systems, we emphasize that PET is not only FDG. Therefore to obtain the most accurate information, tailored for each individual patient, we have to learn and understand the pathophysiological information already provided today by PET in the whole "functional" Universe beyond FDG.

\section{Magnetic resonance imaging}

Although MRI is a morphostructural gold standard, the image is influenced by the molecular environment in which the protons are located, and the technique therefore provides pathophysiological information represented as multiparametric imaging. Consequently enhanced value with respect to $\mathrm{CT}$ is achieved even for structures with similar density but different "biochemical" components, as in soft tissues. Higher sensitivity can be achieved in detecting lesions in the brain, pelvis, and head and neck, and in better defining complex tissue structures, when the differentiator is not the total number of atoms but the whole molecular composition (water, lipids, etc.) and its spatial organization. Although a pathophysiological background is always present, functional MRI (fMRI) procedures analyse biochemistry (through spectroscopy) and/or "dynamic" phenomena with or without the administration of contrast medium.

Because of the lack of ionizing radiation, MRI is an ideal candidate for substituting $\mathrm{CT}$ for morphological imaging in hybrid devices, with the further advantage of having the ability to provide functional information. MRI is based on the interaction between radiowaves and hydrogen nuclei in the presence of a strong external magnetic field. The relaxation times $\mathrm{T} 1$ and $\mathrm{T} 2$, form the basic MR parametric images, although by varying the pulse sequences and sequence times, a wider variety of tissue contrasts tailored for different organs can be obtained [6]. In this sense, also as a consequence of multiparametric imaging, better tissue characterization can be obtained than with CT, for example in demyelinating cerebral diseases or in better staging and restaging of pelvic tumours.

\section{Magnetic resonance spectroscopy (MRS)}

Magnetic resonance spectroscopy (MRS) is based on radiofrequency (RF) detection of signals generated by the magnetic nuclear spin of active nuclei precessing in an external magnetic field after excitation with a pulse transmitted by a RF coil. MRS offers the ability to study biomolecules containing ${ }^{1} \mathrm{H},{ }^{31} \mathrm{P},{ }^{13} \mathrm{C}$ and ${ }^{19} \mathrm{~F}$, and thus may provide enhanced information on the chemical environment, such as number of chemical bonds, neighbouring nuclei and overall chemical structure, leading to the ability to evaluate the pathological metabolic phenotype of cancer cells which show high glucose uptake, increased glycolytic activity and lactate production, decreased mitochondrial activity, low bioenergetic status, and aberrant phospholipid metabolism. In particular, choline phospholipid metabolism is profoundly altered in almost every tumour cell type, which show elevated phosphocholine and increased choline-containing metabolites. Proton or ${ }^{31} \mathrm{P}$ MRS can detect endogenous metabolic changes in vivo, in tumour tissue biopsies and in tumour xenograft models, and has already demonstrated clinical usefulness both in oncological and nononcological diseases. At present MRS is affected by limitations including: (a) overlap of the peaks of similar molecules; (b) spurious changes in the peak of interest may be caused by changes in the normalization term, since measurements are semiquantitative being the ratio of one peak to another; (c) overlay of spectral information with corresponding anatomic images or parametric PET images may be problematic if spectra are derived from a small tissue volume; and (d) the procedure is time consuming and nonpanoramic, analysing a small field of view [7].

\section{Contrast media}

MRI contrast agents shorten the $\mathrm{T} 1$ relaxation time of protons [8]. Gadolinium as a complexed ligand is most commonly used in MR angiography and for brain tumour enhancement associated with alterations in the blood-brain barrier. Gadolinium contrast media with different tissue specificities, such as Gd-BOPTA for MR cholangiopancreatography, are also available. 
Superparamagnetic contrast agents consist of suspended colloids of iron oxide nanoparticles used to reduce the T2 signals of absorbing tissues. Passive targeting strategies rely on superparamagnetic iron oxide (SPIO) uptake in reticuloendothelial cells by receptor-mediated phagocytosis. SPIO is used in liver tumour detection, being concentrated by normal macrophages but not by tumour lesions which appear bright on MRI. Ultrasmall (20-40 nm) SPIO (USPIO) primarily accumulate in the lymph nodes and bone marrow, and are useful in identifying metastatic lymph nodes. Such particles may be applied to track stem cells transplanted into organs or to demonstrate macrophage activity within atherosclerotic plaque [9], and are also a suitable tool to label molecular probes targeting specific tumour-associated markers. Recently, arginine-glycine-aspartic acid USPIO (RGD-USPIO) have been used for in vivo imaging of angiogenesis in xenografts.

\section{Diffusion-weighted imaging (and diffusion tensor imaging)}

Diffusion-weighted imaging (DWI) is a technique exploiting the random, translational motion of water molecules in biological tissues. On diffusion-sensitive sequences, this motion causes phase dispersion of the spins, resulting in signal loss, quantified by calculating the apparent diffusion coefficient (ADC), which refers to the specific diffusion capacity of a biological tissue. Diffusion of water protons in biological tissue derives from the diffusion of extracellular water protons, transport of water protons through the cell membranes, and diffusion of intracellular water protons. The motion is disturbed by intracellular organelles and macromolecules located in the tissue. Accordingly, in viable, densely packed, tumour tissue, water diffusion is obstructed and ADC values are low, while it is higher in normal tissue, and is highest in necrotic areas where water is free to diffuse. Thus, DWI is a potential tool for distinguishing viable tumour from necrosis on the basis of subtle microstructural changes. First applied in diagnosing acute ischaemia, DWI has been proposed for imaging in oncological, inflammatory, infectious and traumatic processes, being an alternative and/or complementary to contrast-enhanced MRI and able to improve sensitivity when added to T2weighted imaging. Because of high lesion-to-background contrast, the additional value of DWI is expected to lie in detection of small lesions unnoticed on anatomical imaging, and promising for early assessment of response to anticancer therapy [10] due to changes in cell density resulting from cytotoxic therapy-induced necrosis or apoptotic processes.

DWI and diffusion tensor imaging (DTI) provide information about orientation, size, integrity and geometry of tissue fibres by measuring the motion of tissue water. In highly organized tissue, such as white matter, water molecules show reduced mobility, and their ADC is therefore lower than in free water. Using DWI and DTI, in the different subtypes of multiple sclerosis there are significant correlations between DTI findings and clinical disability. In addition, DTI allows 'fibre tracking', which is able to generate three-dimensional images of selected axonal tracts. This procedure is useful for investigating individual functional pathways within the brain or cervical spinal cord and in evaluating the remote responses to a demyelinating lesion. Because it allows the encoding of properties that vary with space, shape and orientation of axonal fibres, DTI has the potential to decode these properties and therefore allowing the differentiation of the origin of tissue damage (only myelin or both myelin and axonal injury) [11].

\section{Functional MRI}

The most restrictive definition of fMRI is linked with the blood oxygenation level-dependent (BOLD) response method, commonly employed to reveal activity in the brain. fMRI detects changes induced by cerebral blood flow (CBF) and/or oxygen consumption rate, accompanying modulations in neuronal activity on the regional deoxyhaemoglobin content, acting as an endogenous paramagnetic contrast agent. The information provided is similar to that obtained with FDG PET or radiolabelled water, at least under physiological conditions, whereby glucose metabolism and CBF are coupled [12]; fMRI has replaced PET for activation studies, because of the lack of ionizing radiations and a much greater temporal and spatial resolution. With a leading place in cognitive psychophysiology to investigate local neuronal activation, fMRI may be used to discover networks of activation associated with a variety of pathologies. The BOLD signal reflects vascular variation along with changes in neuronal metabolism. To quantify the relative contribution of the vascular response versus the oxygen extraction fraction and neuronal metabolism, a multimodal model supplementing BOLD with blood volume and $\mathrm{CBF}$ measurements can be used. Cerebral blood volume, $\mathrm{CBF}$ and BOLD data can be used to determine cerebral metabolic rate for $\mathrm{O}_{2}$ and localized neuronal metabolism. All these methods rely on neurovascular coupling to produce a detectable signal. The relationship between the measurable vascular response and the neural signal is termed haemodynamic or neurovascular coupling. This indirect relationship with the desired measurement of neuronal activity will continue to pose challenges, especially in studying interventions and pathologies that are likely to produce or coincide with vascular effects.

In the recent past, whole-body MRI was used almost exclusively in research investigations, either because of long examination times when diagnostic quality sequences were employed, or because of unsatisfactory results when fast 
sequences were preferred. The implementation of a sliding table platform and later the introduction of multireceiver channel MR scanners, using a system of multiple phasedarray coils covering the whole body in the form of a matrix, allowed adequate diagnostic quality, reducing scanning time and creating the technological potential for fused PET and MRI images, and also for whole-body studies [13]. However, a fused image achieved by scans acquired separately may give completely overlapping information with respect to that obtained with simultaneous acquisitions performed with a hybrid machine? Is a hybrid PET/MRI scanner including state of the art PET and MR scanners already available today?

\section{Fusion imaging and hybrid machines}

In analogical diagnostic imaging, morphostructural and functional data are obtained separately, as in a monocular vision with the ability to see only half of a view which can be recomposed by a subjective visual comparison. With the advent of digital imaging so-called fusion imaging has become possible [14]. The images contain functional (PET/ SPECT) or structural (CT/MRI) information, with overlapping data acquired separately. Postprocessing can provide anatomical accuracy that is difficult to obtain due to the problem of reproducibility of positioning in different sessions, and the further disadvantage of the impossibility of measured attenuation correction [15]. The availability of hybrid machines comprising a $\mathrm{CT}$ and a PET (SPECT) scanner working together has allowed the simultaneous acquisition of the two studies with the patient immobile on the same bed, leading to more precise anatomical accuracy with real "binocular vision" of morphostructural and functional data acquired and viewed together. As a further advantage, measured attenuation correction is achievable by $\mathrm{CT}$, allowing more reliable quantitative studies. Furthermore a shorter duration of the diagnostic path is achieved reducing the total cost of the whole diagnostic and/or therapeutic process [16].

Today PET/CT is a primary tool in oncology when using FDG, with new indications in benign diseases and/or with radiotracers beyond FDG. Technological improvement has been obtained with time of flight (TOF) acquisition, together with general progress in both software and hardware allowing, for example, a more strict link with radiotherapy [17]. Nevertheless, some disadvantages cannot be overcome using CT in companion to PET/SPECT in hybrid machines, the first of which is ionizing radiation that, in spite of recent significant dose reductions, represents an insurmountable limitation, mainly when multiple or dynamic scans are requested. Furthermore, low diagnostic accuracy is achieved when a pathology is not associated with significant changes in density, as in demyelinating lesions. Conversely, major advantages with respect to MRI (with comparable spatial resolution) remain the faster time of acquisition and the ability to allow measured attenuation correction in PET/ SPECT studies. Furthermore, hybrid PET/CT scanners are cheaper and technologically more simple than PET/MRI scanners, and are much more widely available with the advantage by a more consolidated know-how.

\section{PET/MRI}

Combining PET and MRI for whole-body studies is challenging, as conventional PET detectors incorporate photomultiplier tubes, which are extremely sensitive to magnetic fields [18]. A previous solution using optical fibres to lead the scintillation light outside the fringe of the magnetic field or at least outside the MRI field of view having been unsatisfactory, a more recent alternative has been to produce PET/MR systems based on compact solid-state silicon photosensors, so-called avalanche photodiodes that are not sensitive to magnetic fields [19].

The ideal PET/MRI system should: (1) have an affordable cost and/or a better cost effectiveness with respect to a PET/CT scanner; (2) be built with the best available PET and MRI technology, allowing fast whole-body and reliable quantitative analysis, including attenuation correction; (3) avoid pitfalls due to movement artefacts and/or to technical issues from misalignment between the PET and MR images; (4) allow the fastest diagnostic course; and (5) allow simultaneous acquisition of PET and MRI data.

At present different commercial strategies have been followed by the three major companies constructing hybrid systems for use in humans, with Philips and GE proposing the closest contiguity between their best PET and MRI scanners, and Siemens already producing a true hybrid machine, allowing the acquisition of a fused image not affected by patient repositioning. More precisely, Philips has developed a system occupying a single room comprising two scanners (TOF PET and 3-T MRI) situated at opposite ends of a turntable in the floor. The MRI study is performed and then the bed turns $180^{\circ}$ and slides the patient into the bore of the PET scanner allowing sequential acquisition allowing later fusion of the data. GE has chosen the "trimodality solution", comprising a top PET/CT scanner, allowing measured attenuation correction, and MRI systems in two adjacent rooms with the patient transferred from one scanner to the other using a dockable table operating as a shuttle. The Siemens true hybrid machine, which is commercially available, allows simultaneous scans, with the PET scanner having new detectors that are not affected by the magnetic field, but does not allow TOF acquisition.

But what do we ask of a PET/MRI system?

1. To do better than PET/CT. 
a. Because of the absence of ionizing radiation, creating clinical indications mainly for paediatric studies and nononcological queries.

b. Because of better ability to evaluate soft tissues and complex pathological components, allowing a more reliable analysis in territories such as the head and neck and pelvis, and/or better definition of tumour recurrences and demyelinating diseases. Conversely, CT is superior for studying lung, bone and calcified lesions.

2. To do as well as PET/CT (or at least not too much worse).

a. Concerning attenuation correction with MRI, further studies are needed to validate present procedures, which already appear to provide reliable attenuation correction at the cerebral level.

b. Whole-body PET/MRI is slower than PET/CT, mainly when a simultaneous PET/MRI scan cannot be acquired.

3. To do what cannot be done by PET/CT: the third eye.

MRI does not depend on differences in density, as does $\mathrm{CT}$, but depends on the expression of a molecular environment that is strictly associated with the living human being; therefore structural information is also dependent on pathophysiology. Furthermore, MRI provides functional information that could represent the greatest enhancement in value when PET and MRI scans are acquired simultaneously. The achievement of this goal at the cerebral level is probably easier because of favourable technical issues and a wider spectrum of functional abilities already expressed by MRI. There could also be an intriguing interaction in whole-body analysis in cancer, for example, connecting the ability of MRI to represent permeability and/or neoangiogenesis overlapping the metabolic patterns imaged by PET. The achievement of "previously unknowable" information could be acquired both by a more strict connection between FDG PET and fMRI studies and by correlating the whole metabolic/functional information achievable with fMRI and PET beyond FDG.

Using a true hybrid fast and accurate PET/MRI scanner, we could open a third eye allowing a new view over the binocular view, based on functional PET imaging fused with morphostructural MRI, further enhanced by the fMRI information, acquired simultaneously.

In spite of the several advantages that the combination of these two powerful techniques may yield, there are potential drawbacks that need to be considered. The strongest negative point is in the wider diffusion of PET/CT, with continuing enlargement of its clinical indications in a diagnostic scenario designed on the basis of evidence-based medicine. The prevalence of PET/CT scanners will be certainly maintained in the near future for economic, technological and cultural reasons, supporting a powerful and cost-effective rationale, further increased by a better analysis of bone and lungs and by contraindications to MRI, including cardiac pacemakers and ferrous metal objects in the patient. Although at the present $\mathrm{CT}$ allows more reliable quantitative analysis, because of measured attenuation correction, absolute quantitation has not yet been achieved. Therefore, comparing "non-absolute" quantitative data, such as SUV, the comparison would only be reliable when the data were obtained using the same machine and identical methodology; for example, values calculated using TOF would not be comparable to those from a traditional PET acquisition.

The need to acquire expertise in reading MRI scans, which involves a learning curve, could be considered a future limitation of PET/MRI. In this regard, active collaboration between nuclear physicians and radiologists would be of pivotal importance, pending the birth of a new more complete expert with the ability to evaluate alone the whole diagnostic content of fused images. Waiting for this growth, that will probably lead to a new vision and division of competences, probably organized on the basis of an organ's pathology more than on technology, nuclear physicians should start to enlarge the width and depth of their background learning, not only relating to the morphostructural universe, but also to the whole functional information achievable by MRI.

Waiting for further technological improvements, it is mandatory to start a cultural revolution, also enlarging horizons outside our specific field in a new world that now seems huge so that we have the ability to derive our knowledge from the revolutionary, complex and not yet readily understood contribution allowed by PET/MRI.

\section{References}

1. Bailey TM, Vealé BL. Advanced computed tomography technology and patient dose: a literature review. Radiol Manage. 2011;33 (5):43-6.

2. Fanti S, Farsad M, Mansi L. PET-CT beyond FDG. Berlin: Springer Verlag; 2010.

3. Rice SL, Roney CA, Daumar P, Lewis JS. The next generation of positron emission tomography radiopharmaceuticals in oncology. Semin Nucl Med. 2011;41(4):265-82.

4. Groves AM, Shastry M, Rodriguez-Justo M, Malhotra A, Endozo R, Davidson T, et al. 18F-FDG PET and biomarkers for tumour angiogenesis in early breast cancer. Eur J Nucl Med Mol Imaging. 2011;38(1):46-52.

5. Arnaldi D, Morbelli S, Morrone E, Campus C, Nobili F. Cognitive impairment in degenerative parkinsonisms: role of radionuclide brain imaging. Q J Nucl Med Mol Imaging. 2012;1:55-67.

6. Negendank W. Studies of human tumors by MRS: a review. NMR Biomed. 1992;5(5):303-24.

7. De Stefano N, Filippi M, Miller D, Pouwels PJ, Rovira A, Gass A, et al. Guidelines for using proton MR spectroscopy in multicenter clinical MS studies. Neurology. 2007;69(20):1942-52.

8. Geraldes CF, Laurent S. Classification and basic properties of contrast agents for magnetic resonance imaging. Contrast Media Mol Imaging. 2009;4(1):1-23. 
9. Modo M, Hoehn M, Bulte JW. Cellular MR imaging. Mol Imaging. 2005;4(3):143-64.

10. Tunariu N, Kaye SB, Desouza NM. Functional imaging: what evidence is there for its utility in clinical trials of targeted therapies? Br J Cancer. 2012;106(4):619-28.

11. Tournier JD, Mori S, Leemans A. Diffusion tensor imaging and beyond. Magn Reson Med. 2011;65(6):1532-56.

12. Abraham T, Feng J. Evolution of brain imaging instrumentation. Semin Nucl Med. 2011;41(3):202-19.

13. Gore JC, Manning HC, Quarles CC, Waddell KW, Yankeelov TE. Magnetic resonance in the era of molecular imaging of cancer. Magn Reson Imaging. 2011;29(5):587-600.

14. Todd-Pokropek A. Advances in computers and image processing with applications in nuclear medicine. Q J Nucl Med. 2002;46(1):62-9.
15. Israel O, Keidar Z, Iosilevsky G, Bettman L, Sachs J, Frenkel A. The fusion of anatomic and physiologic imaging in the management of patients with cancer. Semin Nucl Med. 2001;31(3):191205.

16. Lucignani G, De Palma D. PET/CT in paediatric oncology: clinical usefulness and dosimetric concerns. Eur J Nucl Med Mol Imaging. 2011;38(1):179-84.

17. Bettinardi V, Picchio M, Di Muzio N, Gianolli L, Messa C, Gilardi MC. PET/CT for radiotherapy: image acquisition and data processing. Q J Nucl Med Mol Imaging. 2010;54(5):455-75.

18. Pichler BJ, Judenhofer MS, Wehrl HF. PET/MRI hybrid imaging: devices and initial results. Eur Radiol. 2008;18(6):1077-86.

19. Delso G, Ziegler S. PET/MRI system design. Eur J Nucl Med Mol Imaging. 2009;36 Suppl 1:S86-92. 